\title{
Burocracia local e qualidade da implementação de políticas descentralizadas: uma análise da gestão de recursos federais pelos municípios brasileiros
}

\author{
Mariana Batista ${ }^{1}$ \\ Universidade Federal de Pernambuco
}

A qualidade da burocracia local reduz as falhas na implementação de recursos de transferências federais pelos municípios brasileiros? Este trabalho analisa o processo de implementação de políticas que fazem uso de recursos federais, com foco nas irregularidades identificadas na implementação. Dados do "Programa de Sorteios Públicos" da Controladoria-Geral da União, com amostra aleatória dos municípios brasileiros (20032004), são usados como medida de qualidade de implementação. Como fator explicativo principal, são analisadas quatro medidas da qualidade da burocracia: a inadequação, o quantitativo, a qualificação e a ausência de politização. Com o uso de modelo binomial negativo, os resultados indicam que quanto maior a inadequação da burocracia, maior o número de falhas de implementação e de irregularidades na gestão de recursos federais; e quanto maior o número de funcionários por habitante e a proporção de funcionários com ensino superior, menor o número de falhas e de irregularidades na implementação de recursos federais. Esses resultados indicam a importância da qualidade da burocracia para explicar a implementação de políticas descentralizadas.

Palavras-chave: políticas públicas, implementação, burocracia, descentralização administrativa, receita pública, administração municipal, desigualdade regional

\footnotetext{
1 Agradeço a Francisco Ramos por disponibilizar seu banco de dados sobre as auditorias da Controladoria Geral da União e a Romero Maia pela ajuda com os dados do IBGE. Qualquer incorreção remanescente é de minha total responsabilidade.
}

[Artigo recebido em junho de 2014. Versão final em dezembro de 2014.] 
Burocracia local y calidad de la implementación de políticas descentralizadas: un análisis de la gestión de recursos federales por parte de los municipios brasileños

¿La calidad de la burocracia local disminuye los defectos en la implementación de las transferencias de recursos federales en los municipios? Este trabajo analiza el proceso de implementación de las políticas que hacen uso de los fondos federales, con un enfoque en las irregularidades detectadas en la ejecución. Los datos del "Programa de Sorteios Públicos" de la Contraloría General, con una muestra aleatoria de los municipios brasileños (2003-2004), se utilizan como medida de la calidad de la implementación. Como el principal factor explicativo, se analizan cuatro medidas de calidad de la burocracia: la insuficiencia, el cuantitativo, la cualificación y la ausencia de politización. Utilizando modelos binomiales negativos, los resultados indican que cuanto mayor es la insuficiencia de la burocracia, más fallos de implementación e irregularidades en la gestión de los fondos federales; y cuanto más alto es el número de empleados por habitante y la proporción de trabajadores con educación superior, tanto menor será el número de fallos y irregularidades en la aplicación de los fondos federales. Estos resultados indican la importancia de la calidad de la burocracia para explicar la implementación de las políticas descentralizadas.

Palabras clave: políticas públicas, implementação, burocracia, descentralização administrativa, receita pública, administração municipal, desigualdade regional

\section{Local bureaucracies and the quality of implementation of decentralized policies: an analysis of the management of federal resources by Brazilian municipalities}

Does the quality of the local bureaucracy reduce the failures in the implementation of resources of federal transfers to municipalities? This paper analyzes the process of policy implementation that makes use of federal funds, with a focus on irregularities identified in the implementation. Data from the "Programa de Sorteios Públicos" of the General Controller Office, with a random sample of Brazilian municipalities (2003-2004), are used as a measure of the quality of implementation. As the main explanatory factor four measures of quality of bureaucracy are analyzed: inadequacy, the quantitative, the qualification and absence of politicization. Using negative binomial models, the results indicate that the greater the inadequacy of the bureaucracy, the grater the number of implementation failures and irregularities in the administration of federal transfers and the greater the number of employees per capita and the proportion of employees with higher education, the lower the number of failures and irregularities in the implementation of federal funds. These results indicate the importance of the quality of the bureaucracy to explain the implementation of decentralized policies.

Keywords: public policies, implementation, bureaucracy, administrative decentralization, public revenue, municipal administration, regional inequality 


\section{Introdução}

O Brasil se constitui num sistema federal no qual três níveis de governo são instituídos, com o objetivo expresso de lidar com as especificidades econômicas, sociais e culturais de um grande país. Contudo, para além da distribuição de poder político entre a União, os Estados e os Municípios, o federalismo impõe ainda o problema de distribuição do poder tributário e de prerrogativas de implementação de políticas públicas entre os entes do governo.

Principalmente após a Constituição de 1988, buscou-se uma maior descentralização da arrecadação e também dos gastos no País. Porém, enquanto parte significativa dos tributos ainda é arrecadada no plano federal, uma parcela importante das políticas públicas foi descentralizada para os níveis subnacionais, tendo por justificativa, principalmente, o ganho de eficiência que a maior proximidade entre os decisores e os cidadãos traz em termos de conhecimento das preferências e das necessidades locais (OATES, 1999).

Tendo em vista tal gap vertical de arrecadação entre a União e os entes subnacionais, principalmente os municípios, e também o gap horizontal que se estabelece a partir da diferente capacidade de arrecadação e da desigualdade socioeconômica entre as regiões do País, no federalismo brasileiro foi estabelecido um sistema de transferências intergovernamentais, com vistas a equalizar tais diferenças.

O sistema de transferências intergovernamentais busca estabelecer um padrão mínimo de qualidade na oferta de serviços públicos, independentemente da capacidade de arrecadação da unidade subnacional. Contudo, a questão que se estabelece é se a simples transferência de recursos assegura a implementação de serviços públicos de qualidade ou se a gestão pública local importa. O foco do presente trabalho está na relação entre os diferentes níveis de governo na política de transferência de recursos federais para as localidades. O pressuposto é o de que o impacto dos recursos transferidos depende do processo de implementação no nível local. Isto é, para entender o efeito dos recursos transferidos é preciso analisar a gestão desses recursos pelos governos locais ou o processo por meio do qual os recursos federais são traduzidos em ações entregues à população.

O estágio da implementação de decisões vem sendo considerado o "elo perdido" na análise de políticas públicas (HARGROVE, 1975; FARIA, 2012). Contudo, pode ser considerado um estágio fundamental no que concerne às políticas formuladas no nível federal, mas com implementação descentralizada nos governos locais. Nesse contexto, a perspectiva "de cima para baixo" de implementação de políticas públicas enfatiza a importância da qualidade da burocracia local para a efetiva transição entre a decisão e a entrega dos serviços ao público-alvo. Isto é, para além de quanto é transferido, a qualidade das políticas públicas descentralizadas depende 
diretamente da capacidade da burocracia local de implementar tais recursos. Tendo isso em mente, o objetivo do presente trabalho é analisar o impacto da qualidade da burocracia local sobre a gestão de recursos federais nos municípios brasileiros.

Desde 2003, a Controladoria-Geral da União (CGU) avalia a gestão de recursos de transferências federais para os municípios brasileiros por intermédio de um programa de auditorias por meio de sorteios públicos. Pela análise da implementação dos recursos, são identificados casos de irregularidades na gestão local que podem ser classificados como falha de implementação. O foco aqui é identificar a relação entre a qualidade da burocracia local e a qualidade da implementação de recursos federais. Isto é, a qualidade da burocracia gera melhor gestão de recursos federais?

Para responder tal questionamento, primeiramente o sistema descentralizado de implementação de políticas públicas no Brasil e a importância das transferências federais são analisados. Na seção seguinte, o papel da burocracia na implementação de políticas públicas é sistematizado. Em seguida, a metodologia apresenta os dados, mensuração e modelos estatísticos utilizados. Na quinta seção, são apresentados os resultados da análise do impacto da burocracia local sobre a gestão de recursos federais. Por último, a conclusão.

\section{Federalismo de descentralização de políticas públicas no Brasil}

O Brasil, assim como outras democracias como os Estados Unidos, Suíça, Canadá e Austrália, constitui-se numa Federação. Isto é, estabelece uma organização política em que as atividades de governo são divididas entre os governos regionais e um governo central, de forma que cada unidade do governo tenha autoridade final sobre áreas específicas (RIKER, 1975).

Para além dos fundamentos políticos para o estabelecimento de tal divisão, principalmente relacionados à distribuição e dispersão do poder e à inclusão e respeito às minorias (LIPHART, 2003), o federalismo traz também uma questão econômica diretamente relacionada à distribuição das competências tributárias e de execução das políticas públicas entre os níveis de governo.

No primeiro caso, o argumento geral é o de que o governo central é mais eficiente na arrecadação de certos tributos de base móvel, tendo em vista que permitir que cada membro do Estado estabeleça e recolha livremente seus tributos traria efeitos negativos, como impostos ineficientes e guerra fiscal entre as unidades (OATES, 1999). No segundo caso, o da execução das políticas, um relativo consenso em torno dos efeitos positivos da descentralização se formou no debate político brasileiro na década de 1980 (ARRETCHE, 1996). 
Descentralização significa a transferência de recursos e poder decisório referentes às políticas públicas do nível federal para o nível subnacional, isto é, estados e municípios. No âmbito internacional, esse processo de transferência tomou fôlego nos anos 1980, ficando conhecido como a "revolução da devolução". Os principais benefícios de tal deslocamento estão associados à maior proximidade dos governantes aos cidadãos. Com a maior proximidade, os atores responsáveis pela tomada de decisão têm maior conhecimento das preferências e necessidades dos cidadãos. Adicionalmente, os cidadãos têm maior controle sobre os governantes, aumentando a accountability e a responsabilização. Nesse sentido, a formulação e a implementação de políticas seriam mais eficientes, tendo em vista que incentivam políticas mais bem desenhadas para a localidade onde serão implementadas e também o controle social dos governantes (OATES, 1999; ARRETCHE, 1996; PUTNAM, 2006). Nas palavras de Oates (1999):

A expectativa é que estados e governos locais, estando mais próximos do povo, sejam mais responsivos às preferências do seu eleitorado e sejam mais capazes de encontrar novas e melhores formas de prover os serviços públicos (tradução nossa) (OATES, 1999: 1120)².

O governo central seria mais eficiente na arrecadação de uma parte significativa dos recursos e os governos locais seriam mais eficientes na implementação e acompanhamento de determinadas políticas públicas. Tendo isso em mente, um problema econômico surge: o da distribuição de recursos para a implementação das políticas descentralizadas pelas unidades subnacionais. O Quadro 1 abaixo apresenta a distribuição da arrecadação e do gasto entre os níveis de governo no Brasil.

\section{Quadro 1: Competências de arrecadação e gasto no federalismo brasileiro}

\begin{tabular}{|l|l|l|}
\hline Tributo & Arrecadação & Gasto \\
\hline $\begin{array}{l}\text { Tributos sobre folha de } \\
\text { pagamento }\end{array}$ & União & União, Estados, Municípios, Entidades \\
\hline CPS & União & União \\
\hline FGTS & União & União \\
\hline Salário-educação & União & União, Estados \\
\hline Sistema S & União & Entidades \\
\hline Tributos sobre renda & União & União, Estados, Municípios \\
\hline IR & União & $\begin{array}{l}\text { União (52\%), Estados (21,5\%), Municípios } \\
(23,5)\end{array}$ \\
\hline IOF & União & União \\
\hline
\end{tabular}

2 The hope is that state and local governments, being closer to the people, will be more responsive to the particular preferences of their constituencies and will be able to find new and better ways to provide these services (OATES, 1999, p. 1120). 


\begin{tabular}{|l|l|l|}
\hline Tributo & Arrecadação & Gasto \\
\hline CSLL & União & União \\
\hline $\begin{array}{l}\text { Tributos sobre } \\
\text { consumo }\end{array}$ & $\begin{array}{l}\text { União, Estados, } \\
\text { Municípios }\end{array}$ & União, Estados, Municípios \\
\hline Confins & União & União \\
\hline PIS/Pasep & União & União \\
\hline Cide combustíveis & União & $\begin{array}{l}\text { União (71\%), Estados (21,75\%), Municípios } \\
(7,25 \%)\end{array}$ \\
\hline IPI & União & $\begin{array}{l}\text { União (42\%), Estados (32\%), Municípios } \\
(26 \%)\end{array}$ \\
\hline ICMS & Estados & Estados (75\%), Municípios (25\%) \\
\hline ISS & Municípios & Municípios \\
\hline II & União & União \\
\hline $\begin{array}{l}\text { Tributos sobre } \\
\text { patrimônio }\end{array}$ & $\begin{array}{l}\text { União, Estados, } \\
\text { Municípios }\end{array}$ & União, Estados, Municípios \\
\hline IPTU & Municípios & Municípios \\
\hline IPVA & Estados & Estados (50\%), Municípios (50\%) \\
\hline ITR & União & União (50\%), Municípios (50\%) \\
\hline ITCD & Estados & Estados \\
\hline ITBI & Municípios & Municípios \\
\hline $\begin{array}{l}\text { Taxas e contribuições } \\
\text { de melhoria }\end{array}$ & $\begin{array}{l}\text { União, Estados, } \\
\text { Municípios }\end{array}$ & União, Estados, Municípios \\
\hline Outros tributos & $\begin{array}{l}\text { União, Estados, } \\
\text { Municípios }\end{array}$ & União, Estados, Municípios \\
\hline
\end{tabular}

Fonte: Constituição Federal (2008); Marques, Oliveira e Langemman (2008).

Como pode ser visto no quadro acima, o argumento da distribuição de prerrogativas de tributação entre os níveis de governo é razoavelmente seguido. Contudo, principalmente após a Constituição de 1988, buscou-se uma maior descentralização da arrecadação e também dos gastos no País. Porém, enquanto parte significativa dos tributos ainda é arrecadada no plano federal, uma parcela importante das políticas públicas foi descentralizada para os níveis subnacionais, como pode ser visto na coluna de gasto. O Quadro 2 abaixo apresenta a distribuição de responsabilidades em termos de políticas públicas entre os níveis de governo.

\section{Quadro 2: Distribuição de responsabilidade entre os níveis do governo}

\begin{tabular}{|l|l|}
\hline $\begin{array}{l}\text { Nível de Governo } \\
\text { Responsável }\end{array}$ & Categoria \\
\hline & \\
\hline Federal & \\
\hline & Defesa \\
\hline & Relações exteriores \\
\hline & Comércio internacional \\
\hline & Moeda \\
\hline & Uso da água \\
\hline
\end{tabular}




\begin{tabular}{|c|c|}
\hline $\begin{array}{l}\text { Nível de Governo } \\
\text { Responsável }\end{array}$ & Categoria \\
\hline & Rodovias nacionais \\
\hline & Serviço postal \\
\hline & $\begin{array}{l}\text { Polícia, federal, fronteira, regulação do trabalho, comércio } \\
\text { interestadual, telecomunicações, transporte interestadual, } \\
\text { desenvolvimento urbano, energia, mineração, seguro } \\
\text { desemprego, imigração, cidadania e direitos nativos }\end{array}$ \\
\hline & Segurança social \\
\hline & Sistema nacional de estatística \\
\hline & Regras gerais para sistema nacional de educação \\
\hline \multicolumn{2}{|l|}{ Federal-Estadual } \\
\hline & Saúde \\
\hline & Educação \\
\hline & Cultura \\
\hline & Proteção do meio ambiente e dos recursos naturais \\
\hline & Agricultura \\
\hline & Distribuição de alimentos \\
\hline & Habitação \\
\hline & Saneamento \\
\hline & Bem-estar social \\
\hline & Polícia \\
\hline & Hidroeletricidade \\
\hline \multicolumn{2}{|l|}{ Estadual } \\
\hline & $\begin{array}{l}\text { Responsabilidade residual: assuntos não atribuídos ao nível } \\
\text { federal ou municipal }\end{array}$ \\
\hline \multicolumn{2}{|l|}{ Municipal } \\
\hline & Transporte público (intramunicipal) \\
\hline & Educação básica \\
\hline & Saúde preventiva \\
\hline & Uso da terra \\
\hline & Preservação histórica e cultural \\
\hline
\end{tabular}

Fonte: Constituição Federal (2008); Shah (1990).

Como pode ser visto no Quadro 2 acima, um número expressivo de políticas teve a sua implementação transferida para os governos subnacionais. Especialmente as políticas sociais de educação e saúde passaram a ter a sua base regionalizada. Contudo, um problema se torna premente. Como equacionar o gap vertical de arrecadação entre a União e os entes subnacionais, principalmente os municípios, que passaram a ter novas responsabilidades em termos de políticas públicas, mas não necessariamente possuem os recursos necessários para a sua efetiva implementação.

Adicionalmente a tal gap vertical, há nas federações também o gap horizontal que se estabelece a partir da diferença na capacidade de arrecadação e também da desigualdade socioeconômica entre as regiões do País. Com o objetivo de equilibrar 
as contas da União e dos entes subnacionais e também de equalizar as diferenças regionais, um sistema de transferências intergovernamentais foi estabelecido no País.

Oates(1999) explicaalógica redistributiva dastransferênciasintergovernamentais:

O propósito dessas subvenções é canalizar fundos de jurisdições relativamente ricas para as pobres. Tais transferências são comumente baseadas numa fórmula de equalização que mede a "necessidade fiscal" e a "capacidade fiscal" de cada província, estado ou localidade. Essas fórmulas resultam numa parcela desproporcional das transferências indo para aquelas jurisdições com a mais necessidade fiscal e a menor capacidade fiscal (OATES, 1999: 1127, traduçao nossa) $)^{3}$.

Anualmente, a União transfere para as unidades subnacionais em média $10 \%$ da sua receita (STN, 2011). Os receptores de tais transferências - estados e municípios - são responsáveis pela implementação de parte significativa das políticas sociais, principalmente educação e saúde. Tais transferências assumem um papel de equalização das desigualdades regionais e da busca de um padrão mínimo de qualidade dos serviços públicos em todas as unidades da Federação, independentemente de sua capacidade de arrecadação.

Dito isso, entre a transferência de recursos para a municipalidade e o seu impacto em benefício dos cidadãos locais, problemas de má gestão de recursos podem ocorrer, diminuindo os efeitos positivos da descentralização e o bem-estar da localidade. Nesse sentido, o presente trabalho busca analisar a influência da qualidade da burocracia local sobre a gestão de recursos federais nos municípios brasileiros.

\section{O papel da burocracia na implementação de políticas públicas}

A implementação pode ser considerada o estágio no qual propostas, programas e metas são traduzidas em processos e ações voltadas para a efetiva entrega dos serviços ao público-alvo. Por muito tempo visto como o "elo perdido" na análise de políticas (HARgrove, 1975; FARIA, 2012), esse estágio pode ser um dos mais importantes, já que políticas sem implementação são apenas ideias sem implicações concretas para os cidadãos.

\footnotetext{
3 "The purpose of these grants is to channel funds from relatively wealthy jurisdictions to poorer ones. Such transfers are often based on an equalization formula that measures the "fiscal need" and "fiscal capacity" of each province, state, or locality. These formulas result in a disproportionate share of the transfers going to those jurisdictions with the greatest fiscal need and the least fiscal capacity" (OATES, 1999, p. 1127).
} 
Mas o que exatamente é esse estágio de implementação? Várias definições, filiadas a perspectivas diferentes da implementação, podem ser citadas. No contexto aqui específico da relação entre os entes federativos na implementação de políticas, a definição de Lester e Goggin (1998) parece apropriada. De acordo com os autores, implementação "é um processo, uma série de decisões subnacionais e ações direcionadas no sentido de por a priori decisões federais autoritativas em vigor" (tradução nossa) (LESTER ; GogGIN, 1998, p. 5) 4 .

Fica clara a separação entre formulação e implementação de políticas na definição acima e esse é um dos pontos fundamentais a diferenciar as perspectivas de implementação. As análises da implementação podem ser subdivididas em dois tipos principais: as análises de cima para baixo e as análise de baixo para cima.

A perspectiva de cima para baixo tem como principais características a já citada divisão clara entre a formulação e a implementação de políticas; a hierarquia entre formulação e implementação, estando os formuladores no topo e os implementadores na base; a ideia de déficit de implementação, na qual o desvio do objetivo preestabelecido pode ser mensurado, sendo as principais causas: a baixa qualidade da burocracia, a falha na comunicação entre os níveis e a falha na cooperação entre os níveis (SABATIER, 1986; WINTER, 2010; SABATIER; MAZMANIAN, 1979, 1980; PRESSMAN; WILDAVSKY, 1973).

A perspectiva de baixo para cima critica a centralidade dos formuladores e a visão dos implementadores como "impedimentos", proposta pela abordagem de cima para baixo. A perspectiva propõe o foco nos policy subsystems e não na hierarquia. A implementação gera informação e dá conteúdo à política, de forma que a implementação se torna a formulação em processo. Os chamados burocratas de nível de rua se tornam centrais e as análises passam a ter por foco as relações horizontais em rede no processo de implementação, partindo da ação local e remontando às suas causas (SABATIER, 1986; WINTER, 2010; HEJRN, 1982).

Comparando, a abordagem de cima para baixo propõe um enfoque hierarquizado, separando quem formula e quem implementa, sendo muitas vezes a qualidade dos implementadores variável e fundamental para explicar falhas na entrega de serviços ao público-alvo. Normalmente, tal relação é analisada como um modelo principalagent, buscando identificar os incentivos sobre os implementadores para cooperar com o formulador. Essa separação estrita entre formulação e implementação é bastante criticada pela perspectiva de baixo para cima, propondo um olhar mais amplo sobre o processo de implementação, identificando outros atores e também atividades que mostram o implementador tomando decisões de formulação

\footnotetext{
4 "is a process, a series of subnational decisions and actions directed toward putting a prior authoritative federal
} decision into effect” (LESTER; GOGGIN, 1998, p. 5). 
durante a entrega dos serviços. Contudo, a abordagem de baixo para cima, apesar da riqueza, é menos estruturada e acaba contribuindo pouco para a formulação de teoria (SABATIER, 1986; WINTER, 2010).

Apesar de diferenças fundamentais, algo que é comum às duas abordagens é a centralidade da burocracia no processo de implementação de políticas: "é no processo de implementação dos programas de atuação pública quando é mais clara a influência da burocracia sobre o conteúdo e o alcance de tais programas" (SUBIRATS, 2006, p. 119, tradução nossa) ${ }^{5}$.

A análise aqui proposta tem por base uma perspectiva de cima para baixo da implementação, com foco na burocracia local como implementadora de decisões federais. A estrutura hierárquica do processo decisório, no qual o Governo Federal decide acerca da alocação de recursos federais de transferências voluntárias para os municípios que são responsáveis pela implementação da política e posterior prestação de contas, indica a propriedade da abordagem de cima para baixo. Dessa forma, o governo local se torna o foco da implementação e a sua burocracia, o principal fator a influenciar a qualidade da implementação. Espera-se que a burocracia local afete a implementação de políticas por meio da gestão dos recursos federais.

Quais características da burocracia podem ser consideradas relevantes para a qualidade da implementação? Estudos empíricos apontam como relevantes características o quantitativo de funcionários, a qualificação ou expertise, o comprometimento e também a disposição, analisada como a compreensão da política pelos funcionários, a direção da resposta, se favorável ou contrária, e a intensidade da resposta (PRESSMAN; WILDAVSKY, 1973; SABATIER; MAZMANIAN, 1979, 1980; Van Meter; Van Horn, 1975; SABatier, 1986; Hill; Hupe, 2002).

Num campo da influência política, um debate central é o da politização das agências, ou o uso de indicações políticas para cargos na burocracia. Numa discussão que remonta a Weber, busca-se identificar se funcionários de carreira com regras bem estabelecidas e estabilidade no cargo seriam necessariamente melhores burocratas do que indicados políticos. As indicações políticas têm como justificativa o maior controle político sobre as políticas implementadas, uma vez que funcionários de carreira podem não ter incentivos para agir de acordo com as preferências dos governantes. As indicações podem servir também como uma forma de oxigenar e flexibilizar a máquina pública. Contudo, o uso de tais indicações políticas tem a patronagem como o mais antecipado efeito negativo, quando cargos na estrutura governamental são distribuídos como forma de estabelecer apoio

\footnotetext{
5 "es en el proceso de implementación de los programas de actuación pública cuando resulta más clara la influencia de la burocracia sobre el contenido y el alcance de tales programas" (SUBIRATS, 2006, p. 119).
} 
político. Num dos principais trabalhos empíricos sobre essa longa discussão, Lewis (2007) pergunta-se se indicados políticos de fato implementam piores políticas e chega à conclusão de que burocratas indicados geram resultados inferiores em termos de implementação de políticas do que burocratas de carreira (LEWIS, 2007).

Tendo em mente esse levantamento, o presente trabalho busca avaliar o efeito da qualidade da burocracia sobre a implementação dos recursos de transferências federais. As características da burocracia a serem enfocadas são a inadequação da burocracia, o quantitativo de funcionários, a capacidade e a politização. A hipótese central é de que quanto maior a qualidade da burocracia local, melhor a implementação das políticas que fazem uso de recursos de transferências federais. Os dados e a mensuração das variáveis são apresentados na próxima seção.

\section{Metodologia}

O estudo aqui proposto busca analisar a relação entre qualidade da burocracia local e a implementação de recursos de transferências federais nos municípios brasileiros. O primeiro desafio é quanto à operacionalização de conceitos tão amplos. Como variável dependente, proponho analisar o processo de implementação e não seus produtos ou resultados. Como medida de qualidade da implementação, serão usadas as irregularidades na gestão dos recursos de transferências federais pelos municípios.

A Controladoria-Geral da União (CGU) iniciou, em 2003, um programa de fiscalização com base em sorteios públicos. O programa fiscaliza o uso de recursos de transferências federais pelas unidades subnacionais por meio de auditorias in loco dos municípios sorteados para fiscalização. Na prática, o programa define de forma aleatória municípios que terão suas contas escrutinizadas, com foco nos recursos de transferências federais. Atualmente, a CGU está no 39o sorteio. Em levantamento de 2014, o programa tinha avaliado a implementação de políticas com recursos federais em 2.133 municípios e aproximadamente 21 bilhões de reais ${ }^{6}$.

Fazem parte do sorteio municípios com até 500 mil habitantes e 60 municípios são sorteados a cada sorteio, com exceção dos nove primeiros sorteios que sortearam entre 5 e 50 municípios. Em cada município sorteado, os auditores analisam as contas, obras e serviços que fazem uso de recursos de transferências federais. Ao final, é publicado um relatório com os resultados da auditoria, especificando os programas e as irregularidades encontradas. $\mathrm{O}$ foco da auditoria nos municípios são

\footnotetext{
${ }^{6}$ Informação disponível no sítio da CGU: <http://www.cgu.gov.br/assuntos/auditoria-e-fiscalizacaoavaliacao-deprogramas-de-governo/programa-de-fiscalizacao-por-sorteios-publicos>
} 
os programas implementados no ano da auditoria e nos dois anos anteriores. Os relatórios de avaliação são disponibilizados publicamente no sítio da CGU7?

O banco de dados foi construído com base nesses relatórios públicos de auditoria, consistindo na categorização das irregularidades apresentadas nos relatórios e resultando na redução da grande quantidade de informação qualitativa disponibilizada a uma lista mais restrita de dezenove tipos de irregularidades. A categorização das irregularidades foi realizada por Francisco Ramos, do Departamento de Economia da Universidade Federal de Pernambuco, e sua equipe. O banco de dados está disponível sob solicitação.

O presente artigo analisa os 14 sorteios iniciais realizados entre 2003 e 2004. Trata-se de dados com estrutura cross-section, já que a cada sorteio uma lista diferente de municípios é analisada. Nos 14 sorteios, um total de 710 municípios foi auditorado $^{8}$, identificando 42.874 irregularidades. O Quadro 3 abaixo apresenta a tipologia de irregularidades, num total de 19 tipos. A construção dessa tipologia foi feita com base na classificação de Francisco Ramos.

\section{Quadro 3: Tipologia de irregularidades}

\begin{tabular}{|c|c|}
\hline Superfaturamento & Os preços pagos estão acima do valor de mercado. \\
\hline Licitação & $\begin{array}{l}\text { A licitação apresenta práticas irregulares, como } \\
\text { direcionamento e ausência de concorrência. }\end{array}$ \\
\hline Qualificação & $\begin{array}{l}\text { Qualificação inadequada de pessoal responsável pela } \\
\text { execução do recurso/implementação da política. }\end{array}$ \\
\hline Infraestrutura & Ausência de infraestrutura na execução do recurso. \\
\hline Falha na Contrapartida & $\begin{array}{l}\text { O município não disponibiliza a contrapartida referente ao } \\
\text { município ou a transferência apresenta impropriedades. }\end{array}$ \\
\hline Contrato & Irregularidades no contrato de repasse. \\
\hline Falha Execução & Falha na execução do programa/projeto. \\
\hline Gestão Inadequada & Gestão do bem/obra inadequada. \\
\hline Despesa sem Empenho & Execução de despesas sem empenho. \\
\hline Imposto & Falha no pagamento dos impostos. \\
\hline Prestação de Contas & Falha no processo de prestação de contas. \\
\hline
\end{tabular}

${ }^{7}$ Os relatórios estão disponíveis no seguinte site da CGU: <http://www.cgu.gov.br/Controlelnterno/Avaliacao ExecucaoProgramasGoverno/Sorteios/Municipios/Sorteados/index.asp.>

8 Nos 14 sorteios, foram escolhidos diferentes quantitativos de municípios até estabelecer em 60 municípios. 


\begin{tabular}{|l|l|}
\hline Falha nos Benefícios & $\begin{array}{l}\text { Falha na execução de projetos, acarretando problemas no } \\
\text { repasse dos benefícios. }\end{array}$ \\
\hline Pagamento Indevido & $\begin{array}{l}\text { Forma de pagamento usada indevida, de acordo com a } \\
\text { contratação. }\end{array}$ \\
\hline Reciprocidade & Vinculação nas vendas. \\
\hline $\begin{array}{l}\text { Falha no Conselho/ } \\
\text { Comissão }\end{array}$ & $\begin{array}{l}\text { Falha na atuação do conselho ou da comissão no controle } \\
\text { das atividades previstas no programa. }\end{array}$ \\
\hline Conta Corrente & Falha na administração dos recursos monetários. \\
\hline Liberação & $\begin{array}{l}\text { Falha na comunicação entre os atores para a liberação do } \\
\text { recurso. }\end{array}$ \\
\hline Desperdício & Falha no controle de estoque acarretando desperdício. \\
\hline Sem Classificação & Irregularidades não classificadas. \\
\hline
\end{tabular}

Fonte: Elaboração própria com dados da CGU e Henrique e Ramos (2011).

A tipologia de irregularidades apresentada acima engloba um conjunto bastante diverso de problemas na gestão de recursos federais, desde falhas graves como superfaturamento de obras e indícios de fraudes em licitações, indicando possíveis casos de corrupção, até casos claros de má gestão de recursos como o desperdício, problemas de infraestrutura e falhas na execução do programa. Para lidar com essa heterogeneidade, serão construídas duas especificações diferentes da variável dependente. A primeira refere-se ao número total de irregularidades identificadas no município $i$ no sorteio $t$. Já a segunda especificação restringe as irregularidades ao que pode melhor ser classificado como falha de implementação, isto é, falha na entrega dos serviços ao público-alvo. Nessa segunda especificação, apenas as seguintes irregularidades são consideradas: falha na execução, gestão inadequada, falha nos benefícios e desperdício?

O fator explicativo principal aqui analisado é a qualidade da burocracia. Quatro características da burocracia serão incluídas nos modelos como proxy para qualidade da burocracia. Primeiro, será analisada a ocorrência de irregularidades referentes à inadequação da burocracia a partir da irregularidade "qualificação" apresentada acima. Segundo, será analisado o quantitativo de funcionários sobre o total da população. Terceiro, será analisada a capacitação dos funcionários por meio da escolaridade. Por último, será analisado o efeito da politização por meio do percentual de funcionários que não são estatutários. Os dados têm por fonte a pesquisa Perfil dos Municípios, desenvolvida pelo IBGE.

\footnotetext{
9 Para outras análises que fazem uso dos dados das auditorias da CGU, ver: Albuquerque e Ramos (2006), Ferraz e Finan (2007), Ferraz, Finan e Moreira (2008), Leite (2010), Henrique e Ramos (2011), Melo, Leite e Rocha (2011) e Batista (2013).
} 
Como muitos outros fatores podem influenciar a implementação de recursos federais e não a qualidade da burocracia, será incluída uma série de variáveis de controle. As variáveis são as seguintes: o total de recursos auditorados, o número de ordem do sorteio, se o município foi ressorteado, o IDH, a população e a distância do município para a capital. O total de recursos controla pelo fato de quanto maior o volume de recursos, maior a quantidade de irregularidades. Como o programa de auditorias da CGU é um programa novo, a inclusão do número de ordem nos modelos tenta controlar pelo possível aprendizado da própria CGU. Com o passar dos sorteios, as equipes vão se tornando mais experientes e mais capazes para detectar irregularidades. A variável binária que indica se o município foi sorteado novamente controla pelo efeito restritivo de já ter tido suas contas auditoradas. Espera-se que municípios que já passaram pela auditoria diminuam a quantidade de irregularidades. O índice de desenvolvimento humano controla pelo desenvolvimento do município. Outras variáveis sociais, como escolaridade da população, Gini ou ainda o PIB, não foram incluídas por gerar problemas de multicolinearidade, já que se trata de variáveis altamente correlacionadas com o IDH. A população controla pelo tamanho do município e a distância, pelo possível efeito negativo de estar situada longe da capital. Todas essas são variáveis que podem influenciar o mau desempenho da gestão de recursos federais, que poderiam confundir o efeito da qualidade da burocracia. Dessa forma, é possível minimizar esse problema e tentar isolar o efeito da burocracia sobre a implementação.

Como descrito acima, a variável dependente diz respeito ao número de irregularidades identificadas no município $i$ no sorteio $t$. Como a variável dependente assume apenas valores positivos inteiros, o modelo de regressão mais difundido de mínimos quadrados ordinários não se mostra adequado. 0 modelo adequado é um para dados de contagem. A opção aqui usada é o binomial negativo devido à observação de sobredispersão nos dados (LONG ; FREESE, 2001). A próxima seção apresenta os resultados da análise.

\section{Resultados}

O Gráfico 1 apresenta o quantitativo total de irregularidades por tipo, de acordo com a tipologia apresentada acima. A irregularidade mais frequente é a falha na execução da obra ou projeto, incluindo situações com desvio de finalidade (quando o recurso é utilizado em outra atividade que não a proposta), execução parcial ou não execução completa do programa. É um caso claro de falha de implementação de recursos públicos, quando o valor transferido não é transformado em benefício para a sociedade. A segunda irregularidade mais comum nos municípios analisados é a falha em licitação. Aqui já se trata de uma irregularidade grave e é identificada 
quando há direcionamento na licitação, ausência de concorrência e problemas na documentação ou não elegibilidade de empresas.

Em seguida, aparecem falhas na prestação de contas do uso de recursos de transferências, como documentação irregular e descumprimento do prazo. Gestão inadequada também tem incidência expressiva, indicando casos como subutilização de produtos adquiridos com recursos do programa, obras concluídas, mas sem uso pela população e a não localização de bens e beneficiados do programa. Um exemplo desse último caso ocorre quando, na prestação de contas, consta um programa de treinamento e os beneficiários citados pelo programa não são localizados. Associados também com a gestão inadequada estão casos de desvio de finalidade de produtos, como ônibus escolares que não são usados no transporte de estudantes ou leite que não é distribuído para crianças. Irregularidades no repasse referem-se a problemas de focalização, quando pessoas não elegíveis recebem benefícios, ou duplo recebimento por elegíveis, falhas no cadastramento ou controle de condicionalidades para o recebimento. São falhas associadas a programas de estrutura descentralizada como o Bolsa Família, no qual o município é responsável pela distribuição e pelo controle das condicionalidades.

Gráfico 1: Tipos de irregularidades e incidência nos municípios brasileiros

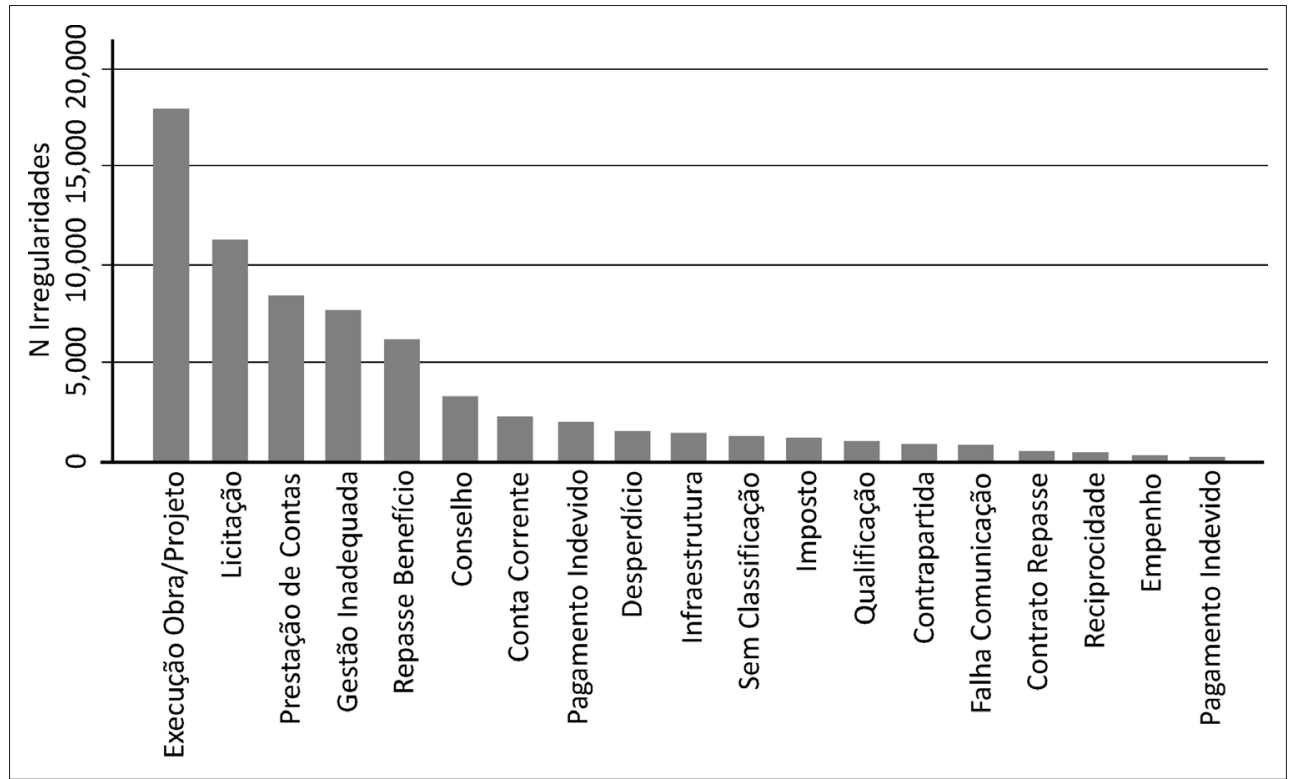

Fonte: Elaboração própria com dados da CGU e Henrique e Ramos (2011). 
Com números menos expressivos, aparecem irregularidades como falha no funcionamento do conselho consultivo, na gestão da conta corrente, pagamento indevido e desperdício (por exemplo, quando remédios perdem a validade antes de serem distribuídos para a população). Por último, as demais irregularidades que apresentam baixa incidência e também irregularidades sem classificação.

O Gráfico 2 apresenta a distribuição das irregularidades por ministério gestor.

\section{Gráfico 2: Incidência de irregularidades por ministério gestor}

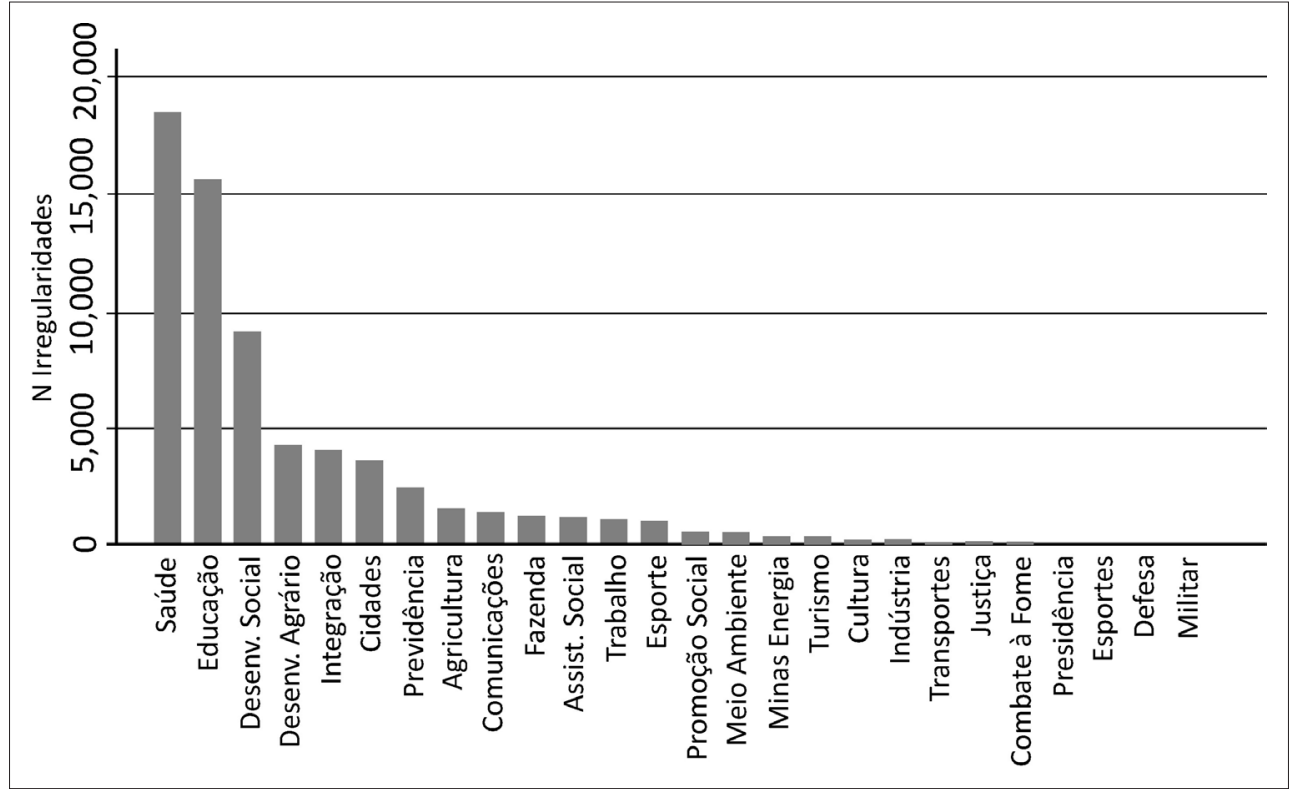

Fonte: Elaboração própria com dados da CGU e Henrique e Ramos (2011).

O Gráfico 2 mostra que os ministérios que apresentam o maior número de irregularidades nos seus repasses são justamente os ministérios da área social: saúde, educação e desenvolvimento social. Como visto na segunda seção deste artigo, a reestruturação das políticas públicas no Brasil pós-Constituição de 1988 privilegiou a implementação descentralizada das políticas sociais. Dessa forma, a maior incidência de irregularidades nesses ministérios não significa que esses ministérios possuem falhas de administração em si, mas apenas que são os ministérios com a maior quantidade de recursos e responsáveis pela maior parte das transferências federais para os municípios.

Para os objetivos desse trabalho, essas irregularidades encontradas pelas auditorias da CGU são consideradas proxy para falhas na implementação de recursos federais pelos municípios brasileiros. Duas medidas alternativas serão utilizadas, ambas contagens. A principal refere-se a uma medida restrita de irregularidades que será aqui chamada de falhas de implementação. Nessa classificação mais 
restrita, estão incluídas as irregularidades falha na execução, gestão inadequada, falha nos benefícios e desperdício. A segunda refere-se ao número total de irregularidades. A utilização de duas medidas diferentes tem como objetivo testar a robustez dos resultados. O total de irregularidades pode ser identificado como falha de implementação, mas é possível que a medida restrita de falha de implementação seja mais homogênea, no sentido de identificar irregularidades que impedem que a política pública seja de fato entregue ao público-alvo.

Como principal fator explicativo das irregularidades encontradas, está a qualidade da burocracia, medida como quatro aspectos diferentes: inadequação, quantitativo, qualificação e carreira. Além das variáveis dependentes e independentes, são incluídas também variáveis de controle. A Tabela 1 abaixo apresenta as estatísticas descritivas das variáveis analisadas.

Tabela 1: Estatísticas descritivas

\begin{tabular}{l|c|c|c|c|c}
\hline Variável & N & Min. & Máx. & Média & Desv. Padrão \\
\hline Burocracia - Inadequação & 710 & 0 & 6 & 0,788 & 1,101 \\
\hline Burocracia - Quantitativo & 705 & 0,005 & 0,170 & 0,033 & 0,016 \\
\hline Burocracia - Qualificação & 688 & 0,001 & 0,527 & 0,111 & 0,087 \\
\hline Burocracia - Carreira & 705 & 0 & 1 & 0,630 & 0,331 \\
\hline Total Recursos & 708 & 0 & 3,12 & 3,22 & 1,42 \\
\hline População & 706 & 1,213 & 454,463 & 25,429 & $39,430.62$ \\
\hline IDH & 703 & 0,479 & 0,919 & 0,692 & 0,080 \\
\hline Distância & 703 & 12,016 & 1,206 & 244.125 & 168.479 \\
\hline
\end{tabular}

Fonte: Elaboração própria com dados da CGU e Henrique e Ramos (2011).

A inadequação da burocracia é medida como o número de irregularidades encontradas pela auditoria da CGU no município analisado, associadas a problemas com a burocracia responsável pelo programa. Essas irregularidades se referem a falta de pessoal ou falta de capacitação para implementação da política. A média é de 0,788 irregularidades por município e desvio-padrão de 1,101.

O quantitativo da burocracia é medido como o número total de funcionários na ativa da administração direta do município sobre a população. Trata-se, então, de uma medida ponderada de funcionários por habitante. A média é de 0,33 funcionários por habitante. A qualificação da burocracia refere-se à proporção de funcionários com ensino superior completo sobre o total de funcionários da administração direta. A média é de 0,11 ou $11 \%$ do total de funcionários concluíram o ensino superior. A variável carreira é uma medida contrária de politização da burocracia. A sua mensuração é a proporção de funcionários estatutários sobre o total de funcionários da administração direta e indica aqueles funcionários que não estão na administração pública apenas por indicação política. A média é de 0,63 ou $63 \%$ dos funcionários têm status estatutário. 
As variáveis de controle são o total de recursos auditorados, a população e o IDH. A média de recursos auditorados é de 32 milhões de reais correntes. A média de habitantes é de 25 mil, com mínimo em 1.213 habitantes e máxima em 454 mil, já que os sorteios são restritos a municípios com menos de 500 mil habitantes. A média do IDH é de 0.692 e a média de distância da capital, de 244 quilômetros.

As variáveis dependentes são o número de falhas de implementação e o número total de irregularidades. A média de falhas de implementação é 31,50, com mínimo de 0 , máximo de 252 e desvio-padrão de 26,12. A média do total de irregularidades é 60,39, com mínimo de 1, máximo de 402 e desvio-padrão de 43,41. 0 Gráfico 3 apresenta a distribuição das variáveis dependentes.

\section{Gráfico 3: Distribuição - Variáveis dependentes}
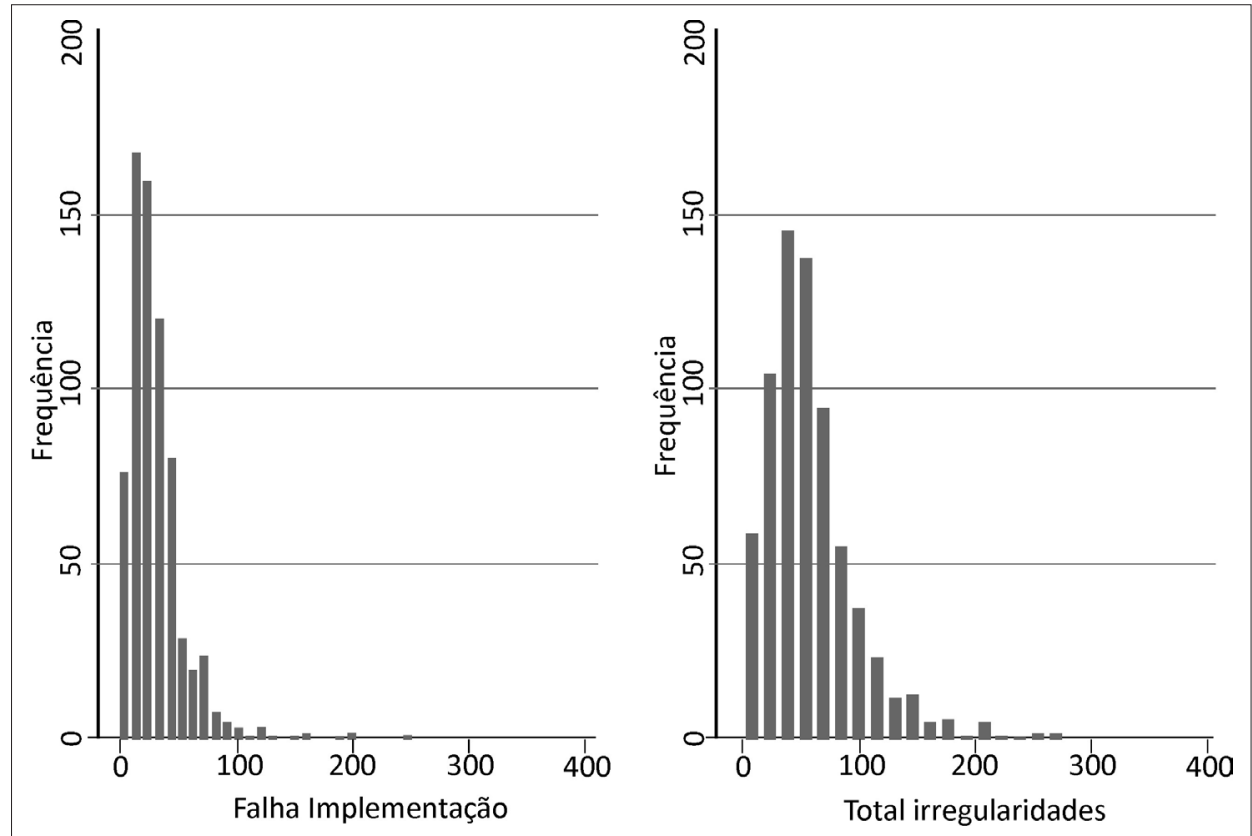

Fonte: Elaboração própria com dados da CGU e Henrique e Ramos (2011).

Os histogramas evidenciam a estrutura de contagem dos dados analisados, assumindo apenas valores positivos inteiros e não apresentando distribuição normal. A Tabela 2 abaixo apresenta os resultados para a análise de regressão com o modelo binomial negativo ${ }^{10}$.

${ }_{10}$ Modelo Binomial Negativo: comando nbreg com erro-padrão robusto, irr no Stata 12. 
Tabela 2: Qualidade da burocracia e implementação de transferências federais

\begin{tabular}{|c|c|c|c|c|}
\hline Variável & Modelo 1 & Modelo 2 & Modelo 3 & Modelo 4 \\
\hline & \multicolumn{2}{|c|}{ VD = N Falha Implementação } & \multicolumn{2}{|c|}{$\mathrm{N}=\mathrm{N}$ Total Irregularidades } \\
\hline Constante & $\begin{array}{c}41.422 * * * \\
(5.543)\end{array}$ & $\begin{array}{c}84.784 * * * \\
(40.920) \\
\end{array}$ & $\begin{array}{c}61.366 * * * \\
(6.375)\end{array}$ & $\begin{array}{c}165.642 * * * \\
(73.551) \\
\end{array}$ \\
\hline $\begin{array}{c}\text { Burocracia } \\
\text { - Inadequação }\end{array}$ & $\begin{array}{c}1.083 * * * \\
(0.033) \\
\end{array}$ & $\begin{array}{c}1.046 \\
(0.031)\end{array}$ & $\begin{array}{c}1.195^{* * * *} \\
(0.030) \\
\end{array}$ & $\begin{array}{c}1.144 * * * \\
(0.026)\end{array}$ \\
\hline $\begin{array}{c}\text { Burocracia } \\
- \text { Quantitativo }\end{array}$ & $\begin{array}{c}0.001^{* * * *} \\
(0.003) \\
\end{array}$ & $\begin{array}{l}0.018 * * \\
(0.037)\end{array}$ & $\begin{array}{c}0.014 * * * \\
(0.024) \\
\end{array}$ & $\begin{array}{l}0.039 * * \\
(0.061)\end{array}$ \\
\hline $\begin{array}{c}\text { Burocracia } \\
\text { - Qualificação }\end{array}$ & $\begin{array}{l}0.445^{* *} \\
(0.163) \\
\end{array}$ & $\begin{array}{l}0.722 \\
(0.176)\end{array}$ & $\begin{array}{l}0.477^{*} \\
(0.207)\end{array}$ & $\begin{array}{l}0.837 \\
10.266\end{array}$ \\
\hline $\begin{array}{l}\text { Burocracia } \\
\text { - Carreira }\end{array}$ & $\begin{array}{c}0.940 \\
(0.109)\end{array}$ & $\begin{array}{c}0.967 \\
(0.098)\end{array}$ & $\begin{array}{c}1.077 \\
(0.127)\end{array}$ & $\begin{array}{l}1.107 \\
(0.115)\end{array}$ \\
\hline Sorteio & - & $\begin{array}{c}1.031 * * * \\
(0.007) \\
\end{array}$ & - & $\begin{array}{c}1.030 * * * \\
(0.006) \\
\end{array}$ \\
\hline $\begin{array}{l}\text { Sorteado } \\
\text { Novamente }\end{array}$ & - & $\begin{array}{c}0.498 * * * \\
(0.125) \\
\end{array}$ & - & $\begin{array}{l}0.610 * * * \\
(0.111) \\
\end{array}$ \\
\hline Total Recursos & - & $\begin{array}{r}1.000 \\
(6.160) \\
\end{array}$ & - & $\begin{array}{l}1.000 \\
(7.330)\end{array}$ \\
\hline População & - & $\begin{array}{c}1.000 * * * \\
(0.100) \\
\end{array}$ & - & $\begin{array}{c}1.000 * * * \\
(0.974) \\
\end{array}$ \\
\hline IDH & - & $\begin{array}{c}0.180 * * * \\
(0.131)\end{array}$ & - & $\begin{array}{c}0.140 * * * \\
(0.096)\end{array}$ \\
\hline Distância & - & $\begin{array}{c}0.999 \\
(0.000)\end{array}$ & - & $\begin{array}{c}0.999 \\
(0.000)\end{array}$ \\
\hline $\begin{array}{c}\text { Log } \\
\text { pseudo-likelihood }\end{array}$ & -3008.2042 & -2952.9122 & -3409.1826 & -3341.2194 \\
\hline Wald chi2 & $16.34 * * *$ & $57.86 * * *$ & $62.73 * * *$ & $138.04^{* * *}$ \\
\hline $\mathrm{N}$ & 688 & 683 & 688 & 683 \\
\hline
\end{tabular}

* sig. a 0,$10 ; * *$ sig. a $0,05, * * *$ sig. a 0,01 .

Incidence rate ratios apresentados. Erro-padrão robusto entre parênteses.

Fonte: Elaboração própria. 
Foram feitos quatro modelos, com as duas especificações da variável dependente - número de falhas de implementação e número total de irregularidades. 0 primeiro modelo foca nas quatro características da burocracia aqui enfatizadas e o modelo seguinte apresenta o modelo completo com as variáveis de controle. Nos quatro modelos, foi incluído erro-padrão robusto para os estados.

Para facilitar a interpretação, são reportadas as incidence rate ratios (irr), que permitem a interpretação direta que não é possível com o coeficiente. Ressaltando que, no caso de irr, valores menores que 1 equivalem a impacto negativo (sinal negativo) e valores maiores que 1 equivalem a impacto positivo (sinal positivo).

Nos quatro modelos, a inadequação da burocracia apresenta o sinal positivo esperado. Isto é, quanto maior o número de irregularidades associadas à inadequação da burocracia, maior o número de falhas de implementação e do total de irregularidades. Nos modelos restritos, apenas com as variáveis de burocracia, o aumento de uma irregularidade de inadequação da burocracia aumenta a falha de implementação a um fator de 1.083. Esse efeito se mantém bastante similar quando a variável dependente é o número total de irregularidades, quando o aumento da inadequação em uma unidade aumenta o total de irregularidades a um fator de 1.195. O resultado se mantém significativo com a inclusão de fatores apenas para o modelo com o total de irregularidades, perdendo significância estatística com a falhas de implementação.

Nos quatro modelos, o quantitativo da burocracia apresenta o sinal negativo esperado e é significativo, indicando um resultado robusto. Isto é, quanto maior a proporção de funcionários por habitantes, menor o número de falhas de implementação e do total de irregularidades. Contudo, o efeito é pequeno, mantendo-se em torno de uma diminuição a um fator de 0.01 .

A terceira especificação da variável burocracia, a qualificação, apresenta o sinal negativo esperado nos quatro modelos, mas atinge significância estatística apenas nos modelos restritos. Isso significa que quanto maior a proporção de funcionários com ensino superior, menor a incidência de falhas de implementação e de irregularidades nas transferências federais para os municípios. Contudo, esse resultado tem que ser lido com cautela, já que não se mantém com a inclusão das variáveis de controle.

Por último, a proporção de estatutários não apresenta resultados significativos em nenhuma das especificações. Esse resultado contraria a expectativa teórica de que a maior proporção de funcionários estatutários, remetendo à menor politização da administração pública, reduziria a ocorrência de falhas de implementação e do total de irregularidades. Esse resultado pode levar a duas interpretações diferentes. Primeiro, não há relação entre politização e qualidade da implementação. Isto é, 
regras e procedimentos da administração pública ou a própria estrutura hierárquica faz com que indicações políticas não afetem o resultado da gestão. Segundo, a especificação da variável pode ser problemática, já que não identifica quais funcionários de fato possuem vinculação partidária. Nesse sentido, mais pesquisas sobre o tópico precisam ser realizadas para identificar se há efeito da politização da burocracia sobre a qualidade da implementação das políticas descentralizadas.

Com relação às variáveis de controle, os resultados mais interessantes são que, de fato, há um aprendizado pela CGU, já que o acúmulo de sorteios aumenta o número de irregularidades encontradas. No modelo 2, o aumento do sorteio aumenta a falha de implementação a um fator de 1.021, resultado que se mantém quando a variável dependente é o total de irregularidades. Os municípios que passam por uma segunda auditoria apresentam número menor de irregularidades nas duas especificações. No modelo 2, o ressorteamento reduz a falha de implementação a um fator de 0.498 , efeito que é maior (0.610) quando a variável dependente é o total de irregularidades. O IDH também afeta negativamente a falha de implementação e o total de irregularidades, indicando que cidades mais desenvolvidas também apresentam melhores resultados na implementação das políticas. O efeito é de 0.180 quando a variável dependente é a falha de implementação e 0.140 quando a variável dependente é o total de irregularidades.

As estimativas dos modelos são apresentadas no final da tabela. O log pseudolikelihood (no lugar do log likelihood) é reportado, uma vez que o erro-padrão robusto com cluster por estado é usado. O Wald indica que os 4 modelos são significativos como um todo.

A interpretação dos resultados, em termos do número predito de eventos, é apresentada no Gráfico 4 abaixo com as diferentes especificações da variável de interesse que apresentaram significância estatística.

O painel superior apresenta os resultados para a falha de implementação, e o painel inferior, para o total de irregularidades. Como mostrado na tabela de regressão, o efeito da inadequação é positivo, enquanto o efeito das demais medidas de qualidade da burocracia é negativo. O efeito, porém, difere na magnitude. Quando há o aumento de um caso de inadequação da burocracia, o número predito de falhas de implementação é 33, alcançando 50 quando o número de casos de inadequação atinge o valor máximo. Esse efeito máximo, quando a variável dependente é o total de irregularidades, é ainda maior, saindo de 50 casos de irregularidade quando a inadequação é 0 , atingindo 150 casos quando a inadequação assume o valor máximo. 
Gráfico 4: Burocracia - quantitativo e número predito de irregularidades

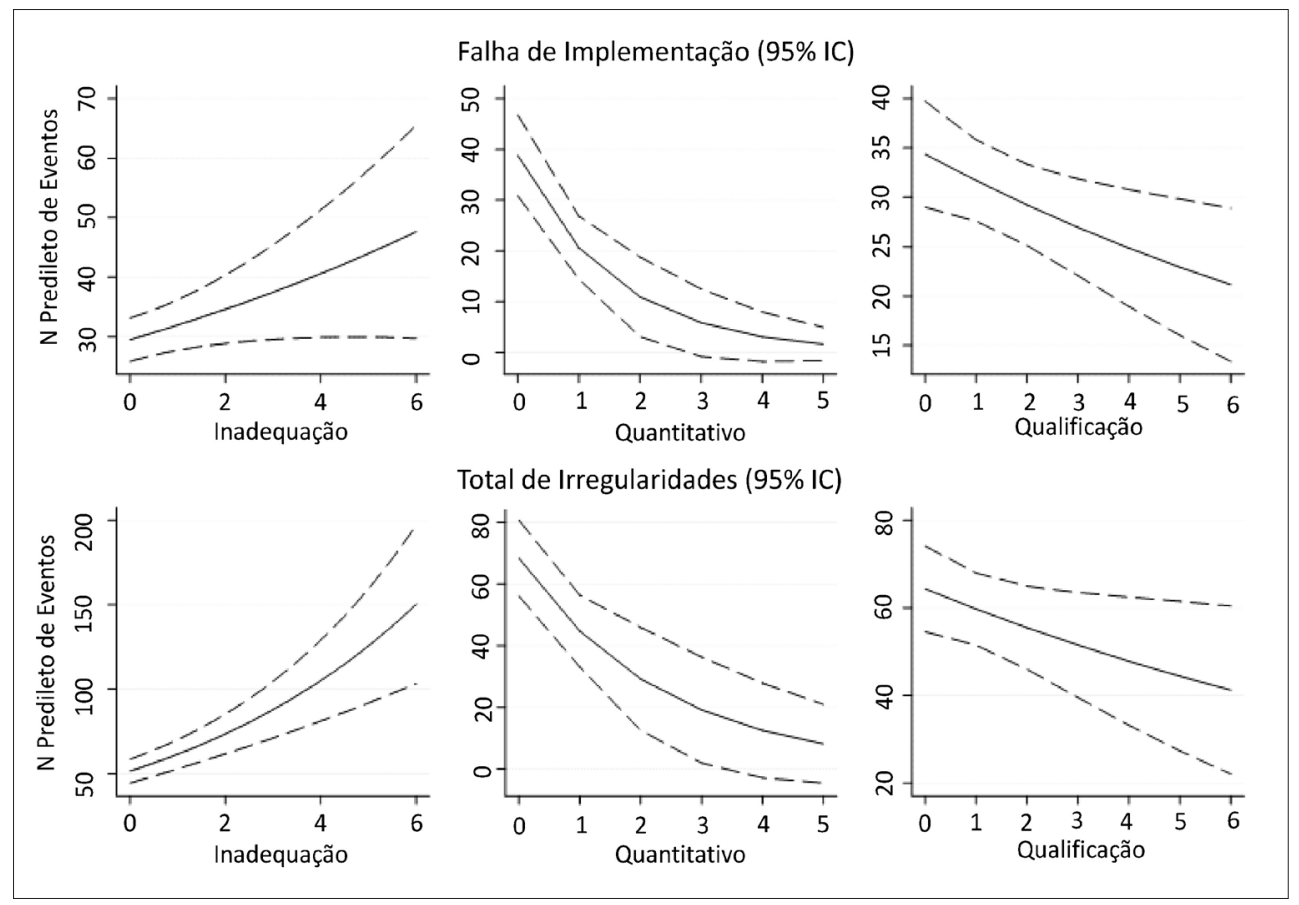

Fonte: Elaboração própria com dados da CGU e Henrique e Ramos (2011).

Sobre o quantitativo da burocracia, quando a proporção de funcionários por habitante é 0 , o número de falhas de implementação é 40 e o número de irregularidades totais é 70. Quando esse número chega ao valor máximo, o número de falhas de implementação tende a 0 e o de irregularidades totais cai para 10. Por último, a qualificação também apresenta efeito negativo sobre a falha de implementação e o total de irregularidades. O número de falhas é 35 quando a proporção de funcionários com ensino superior é 0 e o número total de irregularidades é 62 . O número predito, quando a proporção de funcionários qualificados atinge o valor máximo, é 21 para o número de falhas de implementação e 40 para o total de irregularidades.

Por fim, os resultados aqui apresentados são incipientes, mas indicam um caminho de análise para a relação entre qualidade da burocracia e implementação de políticas públicas descentralizadas no Brasil. Como resultado, pode-se identificar o efeito da inadequação, do quantitativo de funcionários e da sua capacitação sobre a qualidade da implementação. Dessa forma, há indícios de que a qualidade da burocracia está associada à melhor gestão de recursos de transferências federais e, por conseguinte, à entrega de melhores serviços para a população. 


\section{Conclusão}

O presente artigo discutiu a complexidade da implementação de políticas públicas no contexto da descentralização. A necessidade de reduzir as desigualdades regionais e o déficit de arrecadação dos municípios responsáveis pela implementação de políticas fundamentais para a população fez surgir um amplo sistema de transferências federais. Esses recursos de transferências são traduzidos em políticas públicas pelos municípios, transformando a implementação no estágio fundamental. Recursos não necessariamente são traduzidos em serviços eficientes, de forma que a qualidade da implementação se torna determinante.

Um dos aspectos mais enfatizados na literatura sobre implementação é o papel desempenhado pela burocracia para a efetiva implementação das políticas. 0 presente artigo analisou o efeito da qualidade da burocracia sobre a implementação dos recursos de transferências federais nos municípios brasileiros. O foco centrouse nos dados da Controladoria-Geral da União, que identifica irregularidades na implementação de políticas que fazem uso de recursos de transferências federais no período 2003/2004. Duas medidas alternativas da variável dependente foram utilizadas: o número total de irregularidades no município e a medida mais restrita do número de casos de falha de implementação no município.

A qualidade da burocracia foi analisada com base nas principais recomendações da literatura. O conceito foi observado em quatro medidas diferentes: a inadequação da burocracia para a implementação da política em questão, o quantitativo de funcionários, a proporção de funcionários com formação superior como proxy para a qualificação da burocracia e também a proporção de funcionários estatutários como indicativo de ausência de politização. Os resultados alcançados por meio de modelos binomial negativo indicaram que a inadequação aumenta o número de falhas de implementação e o total de irregularidades. O quantitativo de funcionários da burocracia local diminui significativamente o número de falhas e irregularidades na implementação de recursos. Também com resultados expressivos, aparece a proporção de funcionários com ensino superior, indicando que a qualificação da burocracia local reduz problemas na implementação de recursos descentralizados. Por último, a politização da administração não apresentou resultados significativos.

Este trabalho buscou contribuir para a análise do impacto da qualidade da burocracia sobre a implementação de políticas públicas descentralizadas. Algumas questões ainda permanecem em aberto, como, por exemplo, o efeito da politização da burocracia sobre os resultados da gestão pública. Esse tema, tradicional na literatura norte-americana, vem recebendo pouca atenção sistemática no Brasil e pode contribuir bastante para o desenvolvimento da administração pública. 
Espera-se que o desenho de pesquisa aqui proposto e os resultados alcançados contribuam para o desenvolvimento de trabalhos empíricos sobre políticas públicas no Brasil, em especial sobre o estágio da implementação. O desenvolvimento e o acúmulo de outras análises podem contribuir para o aperfeiçoamento da implementação de recursos federais e, por conseguinte, da qualidade das políticas públicas no Brasil.

\section{Referências bibliográficas}

Albuquerque, Breno Emerenciano; Ramos, Francisco. Análise teórica e empírica dos determinantes de corrupção na gestão municipal. In: ENCONTRO NACIONAL DE ECONOMIA - ANPEC, 34, 2006, Salvador, Anais do XXXIV Encontro Nacional ANPEC, Salvador, 2006.

ARRETCHE, Marta. O mito da descentralização: maior democratização e eficiência das políticas públicas? Revista Brasileira de Ciências Sociais, no. 31, 1996.

BATISTA, Mariana. Incentivos da Dinâmica Política sobre a Corrupção: reeleição, competitividade e coalizões nos municípios brasileiros. Revista Brasileira de Ciências Sociais, v. 28, n. 82, 2013.

Controladoria-Geral dA UniÃo (CGU). O Programa de Fiscalização por Sorteios Públicos. Disponível em: <http://www.cgu.gov.br/AreaAuditoriaFiscalizacao ExecucaoProgramasGoverno/Sorteios/leiamais.asp>. Acesso em: 12 jan. 2011.

CONTROLADORIA-GERAL DA UNIÃo (CGU). Relatórios de Fiscalização do 1 o ao 14으 Sorteio do Programa de Fiscalização a partir de Sorteios Públicos. Disponível em: <http://www.cgu.gov.br/sorteios/index2.asp> Acesso em: 12 jan. 2011. 2011b.

BrASIL. Constituição Federal. Constituição da República Federativa do Brasil. 2008. DuARTE, Jose; SILVA, Alexandre; LUz, Everaldo; Gerardo, Jose. Transferências fiscais intergovernamentais no Brasil - avaliação das transferências federais com ênfase no Sistema Único de Saúde.

ELHIRAIKA, Adam. Fiscal decentralization and public service delivery in South Africa. ATPC, Work in Progress, no 58. UNDP.

FARIA, Carlos Aurélio Pimenta. Implementação de Políticas Públicas: teoria e prática. Belo Horizonte: Ed. PUC Minas, 2012.

FERRAZ, Claudio; FINAN, Frederico. Electoral accountability and corruption in local governments: evidence from audit reports. IZA Discussion Paper, no 2843, 2007.

FerraZ, Claudio; FInAN, Frederico; MoreIRA, Diana. Corrupção, má gestão, e desempenho educacional: evidências a partir da fiscalização dos municípios. Preparado para apresentação na ANPEC. 2008.

Freinkman, Lev; PekHANov, Alexander. Fiscal decentralization and the quality of public services in Russian regions. Working Paper, no 111. European Bank for Reconstruction and Development. 2009. 
HARGROVE, Erwin. The missing link: the study of the implementation of social policy. Washington: The Urban Institute, 1975.

Henrique, Angelica; RAmos, Francisco. Corrupção e gastos públicos: um estudo empírico para os municípios de Pernambuco. In: ENCONTRO NACIONAL DE ENGENHARIA DE PRODUÇÃO, 31, 2011, Ubatuba. Anais do XXXI encontro nacional de engenharia de produção. 2011.

HERJN, Benny. Implementation research: the link gone missing. Journal of Public Policy, v. 3, n. 2. 1982.

HILL, Michael; LUPE, Peter. Implementing public policy: governance in theory and practice. London: Sage Publications, 2002.

IBGE. Séries estatísticas e históricas: média anos de estudo, 15 anos ou mais. Disponível em: <http://seriesestatisticas.ibge.gov.br/lista_tema.aspx?op=0\&no=4.> Acessado em: 12 de fevereiro de 2011.

IMF. Disponível em: <http://www.imf.org/external/index.htm.> Acessado em: 12 de fevereiro de 2011.

IPEADATA. Disponível em: <http://www.ipeadata.gov.br/Default.aspx.> Acessado em: 12 de fevereiro de 2011.

LEITE, Adailton. Descentralização, responsabilização e (des)controle: determinantes e impactos da corrupção e má gestão dos recursos federais nos municípios brasileiros. 2010. Tese de Doutorado apresentada no Programa de Pós-Graduação em Ciência Política da Universidade Federal de Pernambuco, Recife .

LESTER, James; GogGIN, Malcom. Back to the future: the rediscovery of implementation studies. Newsletter of the Public Policy Section, American Political Science Association, v. 8, n. 3, 1998.

LEWIS, David. Testing Pendleton's Premise: do political appointees make worse bureaucrats? The Journal of Politics, v. 69, n. 4, 2007.

LIJPHART, Arendt. Modelos de Democracia: desempenho e padrões de governo em 36 países. Rio de Janeiro: Editora Civilização Brasileira, 2003.

LONG, J. Scott; FREESE, Jeremy. Regression models for categorical dependent variables using Stata. Texas: Stata Press, 2001.

MARques JúnIOR, Liderau; Oliveira, Cristiano; LAgemanN, Eugenio. O Jogo da política fiscal entre União, Estados e Municípios no federalismo fiscal brasileiro. Brasília: ESAF, 2008.

MELO, Marcus André; LEITE, Adailton; ROCHA, Enivaldo. Competitive corruption: evidence from randomized Brazilian municipal audits. Paper presented at the ANNUAL MEETING OF THE AMERICAN POLITICAL SCIENCE ASSOCIATION, Seattle 30 August-2 September 2011.

MusgraVE, Richard. The Theory of Public Finance: a study in public economy. New York: McGraw Hill, 1959.

OATES, Wallace. An essay on fiscal federalism. Journal of Economic Literature. v. 37, no 3, 1999. 
PRESSMAN, Jeffrey; WILDAVSKY, Aaron. Implementation. Berkeley: University of California Press, 1973.

PutNAM, Robert. Comunidade e Democracia. Rio de Janeiro: FGV, 2006.

RIKER, William. Federalism. In: GeEnSteIn, Fred; PolSBY, Nelson . Handbook of Political Science, Reading, Mass, 1975.

ROSSI, Peter; LIPSEY, Mark; FREEMAN, Christopher. Evaluation: a systematic approach. California: SAGE Publications, 2004.

SAbatier, Paul; Mazmanian, Daniel. The conditions of effective implementation. Policy Analysis, v. 5, 1979.

SABAtIER, Paul; MAZmanian, Daniel. A framework of analysis. Policy Studies Journal, V. 8, 1980.

SABATIER, Paul. Top-down and bottom-up approaches to implementation research: a critical analysis and suggested synthesis. Journal of Public Policy, n. 6, v. 1, 1986.

$\mathrm{SHAH}$, Anwar. The new fiscal federalism in Brazil. Policy, research and external affairs working paper. World Bank, 1990.

SMITH, Kevin; LARIMER, Christopher. The public policy theory primer. Chicago: Westview Press, 2009.

STN. Tesouro Nacional. Disponível em <http://www.tesouro.fazenda.gov.br/estatistica/ index.asp.> Acessado em: 12 de março de 2011.

SUBIRATS, Joan. El papel de la burocracia en el proceso de determinación e implementación de las políticas públicas. In: SARAIVA, Enrique; FerRAREZI, Elisabete (Orgs). Políticas públicas: Coletânea, v. 2. Brasília, ENAP, 2006.

VAN Meter, Donaldo; VAN HORN, Carl. The policy implementation process: a conceptual framework. Administration and society, v. 6, 1975.

WINTER, Søren. Perspectivas de implementação: status e reconsideração. In: PETERS, B. G. \& PIERRE, J (Orgs). Administração pública: coletânea. São Paulo: Editora UNESP, 2010. 